\title{
ATIC facilitates cell growth and migration by upregulating Myc expression in lung adenocarcinoma
}

\author{
NIU NIU, JIALONG ZENG, XIANNI KE, WENYU ZHENG, CHUNMEI FU, \\ SHIQI LV, JIANGHONG FU and YANG YU
}

\begin{abstract}
Department of Internal Medicine, National Cancer Center/National Clinical Research Center for Cancer/Cancer Hospital and Shenzhen Hospital, Chinese Academy of Medical Sciences and Peking Union Medical College, Shenzhen, Guangdong 518116, P.R. China
\end{abstract}

Received April 16, 2021; Accepted November 30, 2021

DOI: $10.3892 / \mathrm{ol} .2022 .13251$

\begin{abstract}
Aminoimidazole-4-carboxamide ribonucleotide formyltransferase/IMP cyclohydrolase (ATIC), a catalysing enzyme in the de novo purine biosynthetic pathway, has been previously reported to be upregulated and to participate in myeloma and hepatocellular carcinoma progression. In the present study, by using bioinformatics technology, a higher ATIC expression was identified in lung adenocarcinoma (LUAD) tissues than in normal tissues, and ATIC expression was found to be positively associated with Myc expression in LUAD tissues. In addition, the role of ATIC in modulating the growth and migration of LUAD cells was explored and the involvement of Myc was revealed. ATIC expression in 56 paired LUAD and tumour adjacent non-cancerous tissues was assessed using reverse transcription-quantitative PCR and western blot analysis. Pearson's correlation analysis was applied to evaluate the correlation between ATIC and Myc expression levels in LUAD tissues. A rescue experiment was performed to explore the role of ATIC/Myc in regulating the growth, migration and invasion of HCC 827 and NCI-H1435 cells. It was demonstrated that ATIC was overexpressed in LUAD tissues, particularly in advanced-stage LUAD, and was predicted to be associated with an advanced TNM stage, a higher lymph node metastasis rate, poor tissue differentiation and a lower overall survival rate. ATIC overexpression promoted cell growth, migratory and invasive capacities, whereas this effect was abrogated by Myc knockdown in the HCC 827 and NCI-H1435 cells. On the whole, the present study demonstrates that ATIC
\end{abstract}

Correspondence to: Dr Niu Niu, Department of Internal Medicine, National Cancer Center/National Clinical Research Center for Cancer/Cancer Hospital and Shenzhen Hospital, Chinese Academy of Medical Sciences and Peking Union Medical College, 113 Baohe Road, Longgang, Shenzhen, Guangdong 518116, P.R. China E-mail: deanew1025@yeah.net

Key words: 5-aminoimidazole-4-carboxamide ribonucleotide formyltransferase/IMP cyclohydrolase, growth, migration, invasion, Myc promotes LUAD cell growth and migration by increasing Myc expression.

\section{Introduction}

Lung cancer is the leading cause of cancer-related mortality, among which non-small-cell lung cancer (NSCLC) accounts for $\sim 80 \%$ (1). Lung adenocarcinoma (LUAD) and lung squamous cell carcinoma are the two major NSCLC subtypes. The incidence of LUAD has increased in recent years, with the 5-year survival rate value as low as $15 \%$ (2). A number of genes have been reported to be dysregulated and to be involved in LUAD progression; however, the mechanisms underlying the occurrence and development of LUAD remain largely unknown (3-5).

The de novo purine biosynthetic pathway is an energy-intensive pathway with a high conservation, in which inositol monophosphate (IMP) is generated from phosphoribosyl pyrophosphate (PRPP). Accumulating evidence has demonstrated that de novo purine synthesis deregulation may be closely implicated in carcinogenesis (6-8). Cancer cells maintain a high level of de novo purine synthesis, in order to maintain rapid growth (9). 5-Aminoimidazole-4-carboxamide ribonucleotide formyltransferase/IMP cyclohydrolase (ATIC, also known as AICAR) is an enzyme that can catalyse the last two reactions in the de novo purine biosynthetic pathway (10). Recently, it has been reported that ATIC is frequently upregulated and is important for cancer progression and development. For instance, Li et al (11) reported that ATIC was evidently overexpressed in myeloma tissues. Additionally, the aberrant upregulation of ATIC in hepatocellular carcinoma (HCC) tissues, the association of an increased ATIC expression with a poor prognosis, and the suppression of HCC cell viability and migration by ATIC knockdown has been reported (12). However, the role of ATIC in LUAD remains elusive.

Myc is a well-known master transcription factor and modulates the expression of genes that are essential for cell survival, growth and metastasis (13-15). Myc, cyclin D1 (CCND1) and EGFR are the three more frequently amplified oncogenes based on pan-cancer analysis (16). Previous studies have demonstrated that ATIC can activate mTOR signalling, an upstream regulator of Myc $(12,17,18)$. Thus, it was 
hypothesized that ATIC may increase the expression of Myc by activating mTOR signalling.

The present study aimed to explore the role of ATIC in the modulation of cell growth and migration in LUAD, and to further elucidate the role of Myc in this process.

\section{Materials and methods}

Patient tissue samples. LUAD tissues and tumour-adjacent non-cancer tissues were obtained prior to chemoradiotherapy from 56 patients with LUAD post-pneumonectomy, between January, 2013 and January, 2015. The inclusion criteria were as follows: i) Patients with primary LUAD; ii) patients aged from 18 to 75 years; iii) patients or their families signed the informed consent. The exclusion criteria were the following: i) Patients with other types of malignant tumours; ii) patients who received radiation or chemotherapy prior to pneumonectomy. The present study received approval from the Ethics Committee of National Cancer Center/National Clinical Research Center for Cancer/Cancer Hospital and Shenzhen Hospital Chinese Academy of Medical Sciences and Peking Union Medical College prior to the initiation of the study (approval no. KYLX2012-107). Signed written informed consent was acquired by all patients or their families.

Bioinformatics analysis. The gene expression profiling interactive analysis database (GEPIA; http://gepia.cancer-pku. cn/detail.php) was used to analyse the expression of ATIC in LUAD tissues and normal lung tissues. In order to evaluate ATIC expression in LUAD, ATIC was searched for followed by GEPIA. Subsequently, 'Expression DIY' was selected and the 'Boxplot' option was chosen. Finally, the 'LUAD' option was selected in 'Dataset' and the 'Plot' function was implemented.

The StarBase database (http://starbase.sysu.edu.cn/) was used to analyse the association between the expression levels of ATIC and Myc in the LUAD samples. Firstly, the 'Pan-Cancer' option was selected, also closing 'RNA-RNA CoExpression'. Myc was then inserted in the 'Query Gene (lncRNA,mRNA,ncRNA,etc.)' frame and 'ATIC' was inserted in the 'Target Gene (IncRNA,mRNA,ncRNA,etc.)' frame, concurrently selecting 'lung adenocarcinoma' in the 'Cancer' frame, and the 'search' function was used to obtain the final result.

Cells and culture conditions. Three human LUAD cell lines, HCC827 (cat. no. CRL-2868), NCI-H1435 (cat. no. CRL-5870) and HCC4006 (cat. no. CRL-2871), and BEAS-2B cells (human normal lung epithelial cells; cat. no. CRL-9609) were acquired from the American Type Culture Collection (ATCC). HCC827 bears EGFR and TP53 gene mutations, and was initially derived from a 39-year female patient with LUAD. NCI-1435 bears APC, TERT and TP53 mutations, and was initially derived from a 35-year female patient with LUAD. HCC4006 bears an EGFR mutation, and was derived from the pleural effusion of a male patient (aged $>50$ years) with LUAD. All cells were maintained in RPMI-1640 medium with $10 \%$ foetal bovine serum (FBS) and 1\% (v/v) penicillin-streptomycin and incubated at $37^{\circ} \mathrm{C}$ in an atmosphere containing $5 \% \mathrm{CO}_{2}$. The cell culture medium, FBS and penicillin-streptomycin reagents were purchased from Thermo Fisher Scientific, Inc.
Additionally, the cells were treated with $100 \mathrm{nmol} / 1$ rapamycin (Selleckchem) for $2 \mathrm{~h}$ prior to plasmid transfection.

Lentivirus infection and plasmid transfection. The shRNAs used to downregulate ATIC (sh-ATIC-1/2) and Myc (sh-Myc-1/2) expression and the negative control vectors (sh-NC) were obtained from Shanghai GenePharma Co., Ltd., using the lentiviral interfering vector LV-2 (pGLVU6/Puro) (cat. no. C06002, Shanghai GenePharma Co., Ltd.), containing an ATIC- or Myc-targeting shRNA sequence. For lentiviral packaging, the expression vector (pGLVU6/Puro, $20 \mathrm{mg}$ ) and packaging vectors $(1 \mu \mathrm{g} / \mathrm{ml}$; pHelper 1.0 and pHelper 2.0) were transfected into 293T cells (cat. no. CRL-3216; ATCC) using Lipofectamine ${ }^{\circledR} 2000$ (Thermo Fisher Scientific, Inc.), based on the manufacturer's instructions. Following a 48-h culture, supernatants containing sh-ATIC/Myc and sh-NC were harvested, respectively. Purification was then performed using ultracentrifugation at $1,000 \mathrm{x} \mathrm{g}$ and $4^{\circ} \mathrm{C}$ for $2 \mathrm{~min}$ (Himac CT15RE; Hitachi, Ltd.) and the lentiviral titre was determined. For lentiviral infection, a total of $2 \times 10^{5}$ cells were seeded into each well of a 6 -well plate and cultured at $37^{\circ} \mathrm{C}$ overnight. The following day, the cells were infected with the sh-ATIC-1/2, sh-Myc-1/2, or sh-NC lentiviruses (Shanghai GenePharma Co., Ltd.) at multiplicity of infection (MOI) of 5 with the help of polybrene $(6 \mu \mathrm{g} / \mathrm{ml})$. The 3rd generation system was used in following experiments.

The ATIC overexpression vector (vector-ATIC; cat. no. RC203490, OriGene Technologies, Inc.) established in pCMV6-Entry vector was used to upregulate ATIC expression. LUAD cells were transfected with vector-ATIC using Lipofectamine $3000{ }^{\circledR}$ (Invitrogen; Thermo Fischer Scientific, Inc.), according to the manufacturer's instructions. Vector-NC (cat. no. PS100001, OriGene Technologies, Inc.) was used as a negative control for vector-ATIC. Following 48 h of transfection, the cells were harvested for transfection efficiency analysis. The shRNA sequences are designed used using the website of Thermo Fisher Scientific, Inc. (https://rnaidesigner.thermofisher.com/rnaiexpress/setOption.do?designOp tion=shrna\&pid=3449414797065297167). First, 'shRNA' was selected in 'Target Design Options', the 'Accession number' for ATIC (NM_004044.7) and Myc (NM_002467.6), were then inserted, followed by the 'RNAi Design' function. Subsequently, two target sequences with high Rank star rating we selected and the function 'Design shRNA Oligos' was applied. 'CTCGAGC' was then inserted in the 'Custom Loop Sequence', for the acquisition of the shRNA sequences. The shRNA sequences are listed as follows: sh-ATIC-1 forward, 5'-CACCGGTTTGAATCTGGTCGCTTCCCTCGAGGG AAGCGACCAGATTCAAACC-3' and reverse, 5'-AAAAGG TTTGAATCTGGTCGCTTCCCTCGAGGGAAGCGACCA GATTCAAACC-3'; sh-ATIC-2 forward, 5'-CACCGCGTA TCTCAGATGCCCTTGACTCGAGTCAAGGGCATCTGA GATACGC-3' and reverse, 5'-AAAAGCGTATCTCAGATG CCCTTGACTCGAGTCAAGGGCATCTGAGATACGC-3'; sh-Myc-1 forward, 5'-CACCGCTTCACCAACAGGAACTAT GCTCGAGCATAGTTCCTGTTGGTGAAGC-3' and reverse, 5'-AAAAGCTTCACCAACAGGAACTATGCTCGAGCA TAGTTCCTGTTGGTGAAGC-3'; sh-Myc-2 forward, 5'-CAC CGGAAACGACGAGAACAGTTGA/TCAACTGTTCTCGTC GTTTCC-3' and reverse, 5'-AAAAGGAAACGACGAGAA CAGTTGACTCGAGTCAACTGTTCTCGTCGTTTCC-3'. 
Table I. Sequences of primers used for RT-qPCR.

\begin{tabular}{lcc}
\hline Gene & Forward $\left(5^{\prime}-3^{\prime}\right)$ & Reverse $\left(5^{\prime}-3^{\prime}\right)$ \\
\hline ATIC & CGGCCAGCTCGCCTTATTTA & ATTTGCTCCACAGCCTCCTC \\
Myc & GCAATGCGTTGCTGGGTTAT & CGCATCCTTGTCCTGTGAGT \\
$\beta$-actin & CTCGCCTTTGCCGATCC & TTCTCCATGTCGTCCCAGTT
\end{tabular}

ATIC, 5-aminoimidazole-4-carboxamide ribonucleotide formyltransferase/IMP cyclohydrolase; RT-qPCR, reverse transcription-quantitative PCR.

Reverse transcription-quantitative PCR (RT-qPCR). Total RNA was isolated from the tissues and cells (HCC827, NCI-H1435, HCC4006 and BEAS-2) using TRIzol ${ }^{\circledR}$ reagent (Invitrogen; Thermo Fischer Scientific, Inc.) and then subjected to cDNA synthesis using the PrimeScript RT Master Mix kit (RR036A; Takara Bio, Inc.) for $15 \mathrm{~min}$ at $37^{\circ} \mathrm{C}$ and $5 \mathrm{sec}$ at $85^{\circ} \mathrm{C}$. Subsequently, cDNA samples were used for PCR detection with 2X SYBR-Green PCR Mastermix (Beijing Solarbio Science \& Technology Co., Ltd.) on a 7500 Real-Time PCR System (Applied Biosystems; Thermo Fisher Scientific, Inc.). The reactions were carried out as follows: $95^{\circ} \mathrm{C}$ for $1 \mathrm{~min}$, followed by 39 cycles of $95^{\circ} \mathrm{C}$ for $15 \mathrm{sec}$ and $60^{\circ} \mathrm{C}$ for 1 min. $\beta$-actin was used as the reference gene. Relative mRNA expression was calculated based on the $2^{-\Delta \Delta C q}$ method (19). The sequences of the primers used are presented in Table I.

Western blot analysis. Total protein samples were obtained from tissues and cells using RIPA lysis buffer (Beijing Solarbio Science \& Technology Co., Ltd.) supplemented with $1 \%$ protease inhibitor (Beijing Solarbio Science \& Technology Co., Ltd.). Following centrifugation at $4{ }^{\circ} \mathrm{C}$ for $30 \mathrm{~min}$ at a speed of at $12,000 \mathrm{x} \mathrm{g}$, a bicinchoninic acid protein assay kit (Thermo Fisher Scientific, Inc.) was used to define the protein concentrations, according to the manufacturer's specifications. A total of $20 \mu \mathrm{g}$ of protein from each sample were then loaded to a $10 \%$ SDS-polyacrylamide gel, subjected to electrophoresis and transferred to polyvinylidene difluoride membranes (PVDF; MilliporeSigma). The membranes were then probed with primary antibodies overnight at $4^{\circ} \mathrm{C}$, followed by blocking with $5 \%$ non-fat milk for $1 \mathrm{~h}$ at room temperature. The primary antibodies used included an anti- $\beta$-actin antibody (1:5,000 dilution; cat. no. ab8226; Abcam), an anti-ATIC antibody (1:3,000 dilution; cat. no. ab33520, Abcam), an anti-mTOR antibody (1:1,000 dilution; cat. no. 2972, Cell Signalling Technology, Inc.), an anti-phosphorylated (p-)mTOR (p-mTOR) antibody (1:1,000 dilution; cat. no. 5536, Cell Signalling Technology, Inc.) and an anti-Myc antibody (1:2,500 dilution; cat. no. ab32072, Abcam). Subsequently, all membranes were probed with HRP-conjugated goat anti-rabbit IgG (cat. no. ab7090; Abcam) and goat anti-mouse IgG (cat. no. ab97040; Abcam) second antibodies (1:10,000 dilution) at room temperature for $1 \mathrm{~h}$. Following incubation with ECL reagent (MilliporeSigma) for $30 \mathrm{sec}$ at room temperature, the protein signals were measured using ProfiBlot-48 (Tecan Group, Ltd.) and quantified using ImageJ v2.1.4.7 (National Institutes of Health).
CCK-8 assay. LUAD cells $(3,000)$ were placed into each well of a 96-well plate. Cell transfection was then carried out following cell adherence. The cell culture medium was replaced with $10 \mu \mathrm{l}$ CCK-8 solution (Abcam) and $90 \mu \mathrm{l}$ fresh culture medium after 1, 2, 3, 4 and 5 days of cell transfection. Following a 3-h incubation with CCK-8 solution at $37^{\circ} \mathrm{C}$, the OD values $(450 \mathrm{~nm})$ were examined using a spectrophotometer (Thermo Fisher Scientific, Inc.).

Wound healing assay. LUAD cells at a density of $1 \times 10^{6}$ cells/well were seeded in 6-well plates and incubated at $37^{\circ} \mathrm{C}$ overnight. Pipette tips were used for wound formation when the cell confluency reached $100 \%$. In addition, the culture medium was replaced with FBS-free medium. The width was measured using Image-Pro Plus software 6.0 (Media Cybernetics, Inc.) at 0 and $24 \mathrm{~h}$ following wound formation. The cell migration rate was calculated as follows: Cell migration $(\%)=\left(1-\right.$ width $_{24 \mathrm{~h}} /$ width $\left._{0 \mathrm{~h}}\right) \times 100 \%$

Transwell chamber assay. Matrigel (25 $\mu 1$ Matrigel was diluted in $25 \mu \mathrm{l}$ serum-free medium and cultured at $37^{\circ} \mathrm{C}$ for 2 h)-coated Transwell chambers (BD Biosciences) were used for cell invasion assessment. A total of $1 \times 10^{5}$ LUAD cells resuspended in FBS-free medium were placed into the top chamber, while $600 \mu \mathrm{l}$ of culture medium containing 15\% FBS were added into the bottom chamber. Following incubation at $37^{\circ} \mathrm{C}$ for $48 \mathrm{~h}$, cells on the top of the filter were removed using cotton swabs, and cells on the bottom were fixed in $4 \%$ paraformaldehyde and then stained with $1 \%$ crystal violet (Beijing Solarbio Science \& Technology Co., Ltd.) for $10 \mathrm{~min}$ at room temperature. The invaded cells that were removed from the bottom of the chambers were counted under an inverted microscope (BX-42; Olympus Corporation).

Statistical analysis. Three independent experiments were performed for all protocols used in the present study. Statistical analysis was performed by using SPSS (version 23.0; IBM Corp.). The paired Student's t-test was used to compare data between the cancer group and para-carcinoma groups, while the unpaired Student's t-test was used for other data comparisons between two groups. The one-way ANOVA followed by Tukey's post hoc test were used for the data analysis of two groups and multiple groups, respectively. Kaplan-Meier curves with log-rank tests were used to analyse the value of ATIC in predicting the overall survival rates of patients with LUAD. An ATIC expression greater than the average mRNA expression level from the RT-qPCR result was considered a 
Table II. Association of ATIC expression with the clinicopathological features of patients with LUAD.

\begin{tabular}{|c|c|c|c|c|}
\hline \multirow[b]{2}{*}{ Characteristic } & \multirow[b]{2}{*}{ Total no. of patients $(n=56)$} & \multicolumn{2}{|c|}{ ATIC expression } & \multirow[b]{2}{*}{ P-value } \\
\hline & & Low $(n=30)$ & High $(n=26)$ & \\
\hline Age (years) & & & & 0.423 \\
\hline$\leq 60$ & 32 & 18 & 14 & \\
\hline$>60$ & 24 & 12 & 12 & \\
\hline Sex & & & & 0.211 \\
\hline Male & 28 & 13 & 15 & \\
\hline Female & 28 & 17 & 11 & \\
\hline TNM stage & & & & 0.031 \\
\hline I-II & 42 & 26 & 16 & \\
\hline III-IV & 14 & 4 & 10 & \\
\hline Tumour differentiation & & & & 0.035 \\
\hline Well & 34 & 22 & 12 & \\
\hline Moderate-poor & 22 & 8 & 14 & \\
\hline Lymph node metastasis & & & & 0.030 \\
\hline No & 28 & 19 & 9 & \\
\hline Yes & 28 & 11 & 17 & \\
\hline Smoking & & & & 0.274 \\
\hline Smokers & 46 & 26 & 20 & \\
\hline Never & 10 & 4 & 6 & \\
\hline
\end{tabular}

ATIC, 5-aminoimidazole-4-carboxamide ribonucleotide formyltransferase/IMP cyclohydrolase; LUAD, lung adenocarcinoma.

high expression, and a less than or average mRNA expression was considered a low expression. Pearson's correlation analysis was applied to examine the correlation between the expression levels of ATIC and Myc in the LUAD cases. Fisher's analysis was used for data comparisons in Table II. A value of $\mathrm{P}<0.05$ was considered to indicate a statistically significant difference.

\section{Results}

ATIC expression is elevated in LUAD. From the GEPIA database analysis, it was revealed that the ATIC levels were upregulated in the LUAD tissues, as compared with the normal lung tissues (Fig. 1A). Additionally, a 1-fold increase in the expression of ATIC in the 56 paired LUAD tissues was observed, as compared with the tumour-adjacent normal tissues using RT-qPCR (Fig. 1B) and in 3 paired LUAD tissues and the tumour-adjacent normal tissues using western blot analysis (Fig. 1C). In addition, the expression levels of ATIC in three human LUAD cell lines (HCC827, NCI-H1435 and HCC4006) and one human normal lung epithelial cell line (BEAS-2B) were compared. Consistently, the results demonstrated that ATIC expression in the LUAD cells was $\sim 2-3$-fold higher than that in the BEAS-2B cells (Fig. 1D and E). These results demonstrated that ATIC expression was elevated in LUAD tissues and cell lines.

High expression of ATIC is a predictor of an advanced tumour stage and lower survival rates in patients with LUAD. Subsequently, the expression levels of ATIC in the different stages of LUAD were assessed using the GEPIA database. The results revealed that the ATIC level showed significant difference between different stages of LUAD (Fig. 2A). In addition, the association between the ATIC expression levels and the clinicopathological features of patients with LUAD was evaluated. It was demonstrated that ATIC expression was positively associated with the TNM stage and lymph node metastasis, and that the high expression of ATIC was a predictor of a poorer tissue differentiation (Table II). In addition, the 5-year overall survival rate of patients with LUAD with a high ATIC expression was lower than that of patients with a low ATIC expression in the 56 clinical cases of LUAD examined (Fig. 2B). These results clearly demonstrated that a high ATIC expression was associated with an advanced stage and lower survival rates of patients with LUAD.

ATIC promotes cell growth and migration in LUAD. Subsequently, both gain- and loss-of-function assays were performed, in order to explore the role of ATIC in modulating LUAD cell growth and migration. The HCC827 and NCI-H1435 cells exhibited moderate ATIC expression levels in comparison with the HCC4006 and BEAS-2B cells; thus, these two cell lines were used in the following experiments. The expression of ATIC was observed to be decreased by $\sim 50-60 \%$ at the mRNA level and by $70-80 \%$ at the protein level following transfection of the HCC827 and NCI-H1435 cells with sh-ATIC-1 and sh-ATIC-2 lentiviral vectors (Fig. S1A and B), whereas ATIC expression was increased when the cells were transfected with vector-ATIC (Fig. 1C and D). sh-ATIC-2 was used in the following assay, due to 
A

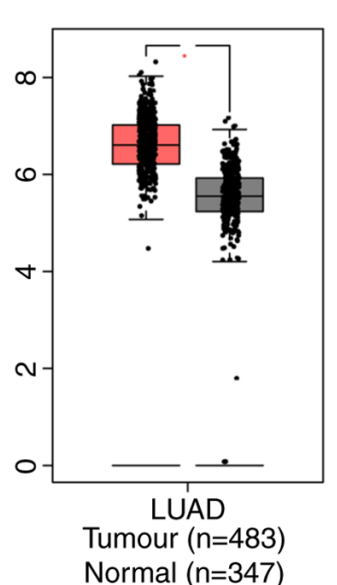

D

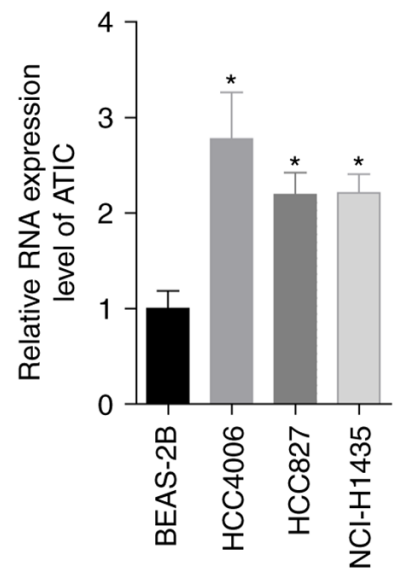

C

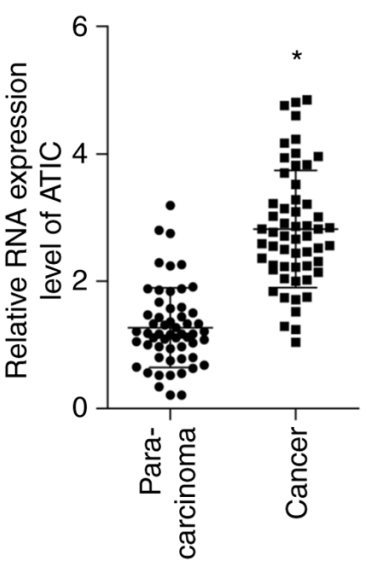

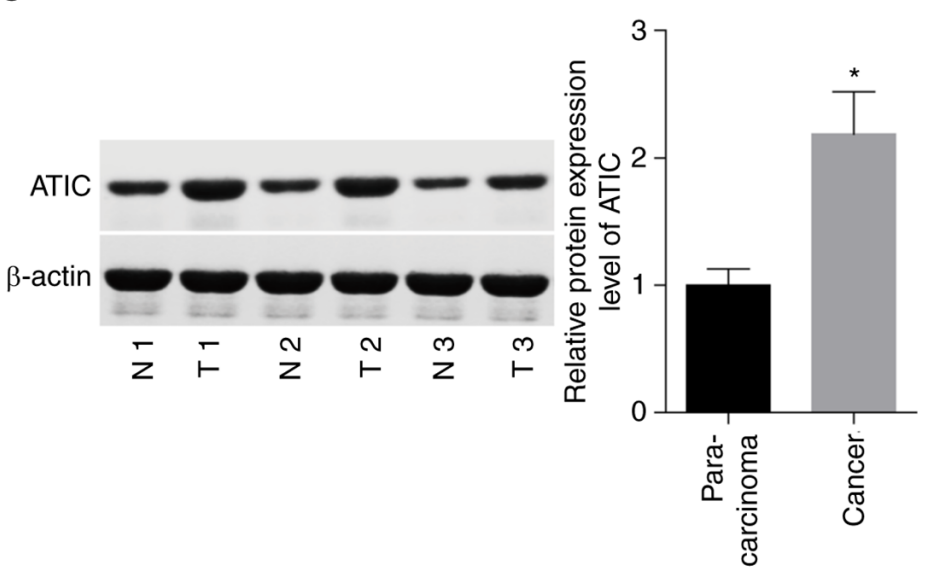

$\mathrm{E}$

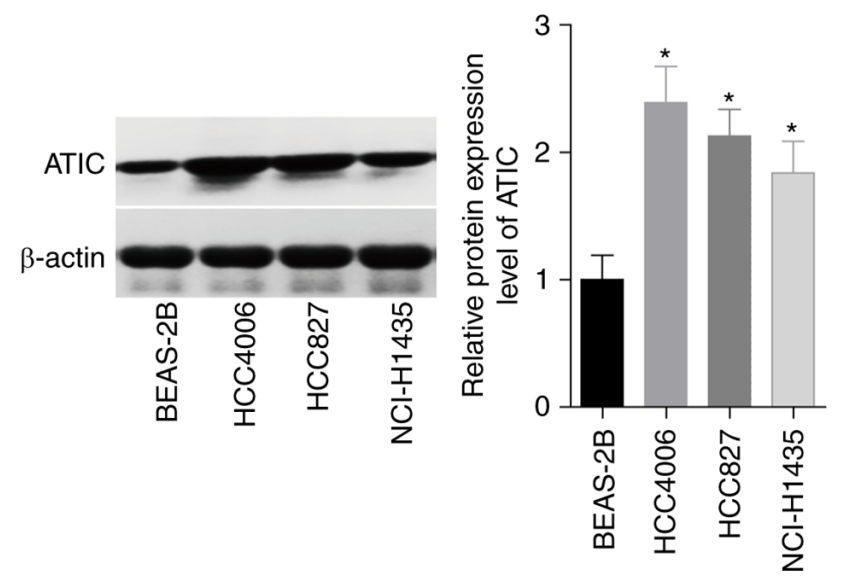

Figure 1. ATIC expression is elevated in LUAD. (A) The GEPIA database was used to analyse the different expression levels of ATIC in LUAD and normal tissues (the red colour represents tumour tissue and the grey colour represents normal tissue; ${ }^{*} \mathrm{P}<0.05$ vs. normal. (B and C) RT-qPCR (56 paired LUAD tissues) and western blot analysis were applied for the detection of ATIC expression in LUAD tissues and paired normal tissues (T, tumour tissues; $\mathrm{N}$, tumour-adjacent normal tissues). (D and E) ATIC levels in HCC827, NCI-H1435, HCC4006 and BEAS-2B cells were determined using RT-qPCR and western blot analysis. ${ }^{*} \mathrm{P}<0.05$ vs. para-carcinoma tissue or BEAS-2B cells. ATIC, 5-aminoimidazole-4-carboxamide ribonucleotide formyltransferase/IMP cyclohydrolase; LUAD, lung adenocarcinoma; GEPIA, gene expression profiling interactive analysis; RT-qPCR, reverse transcription-quantitative PCR.

A

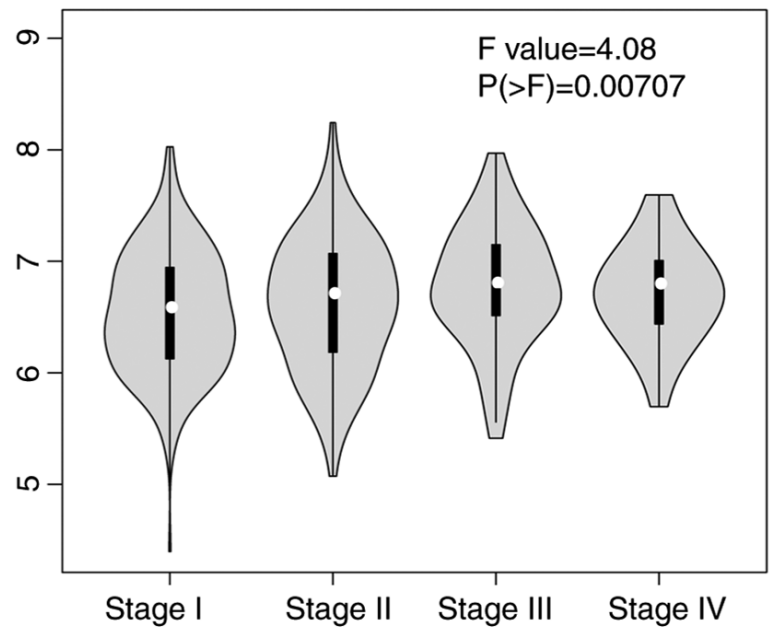

B

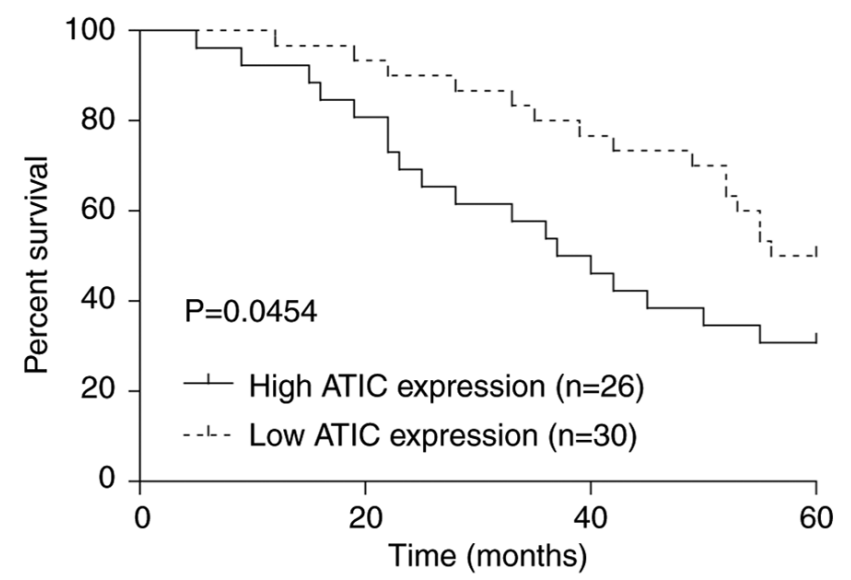

Figure 2. A high expression of ATIC is a predictor of an advanced stage and a lower survival time. (A) ATIC levels in LUAD tissues with different clinical stages were analysed using the GEPIA database. (B) Kaplan-Meier curves with log-rank tests were used to analyse the value of ATIC in predicting patients' overall survival time in LUAD. ATIC, 5-aminoimidazole-4-carboxamide ribonucleotide formyltransferase/IMP cyclohydrolase; LUAD, lung adenocarcinoma; GEPIA, gene expression profiling interactive analysis. 
A

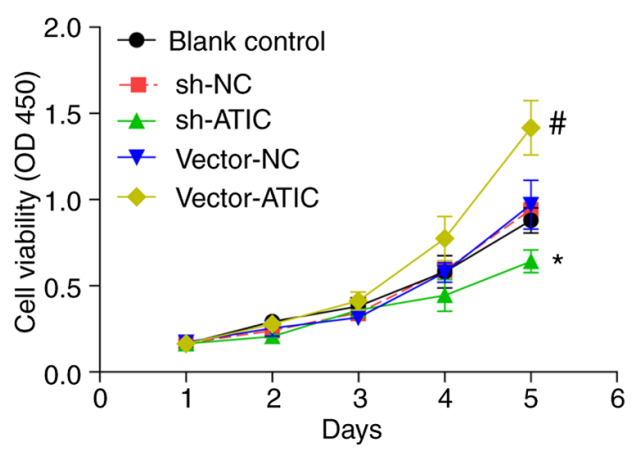

C

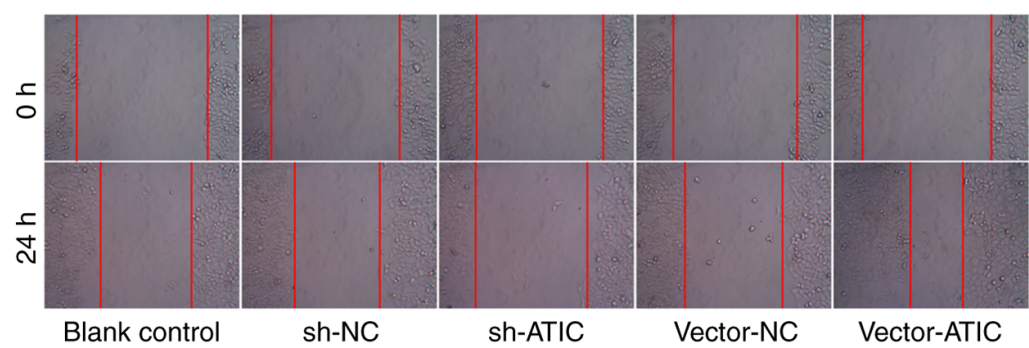

$\mathrm{D}$

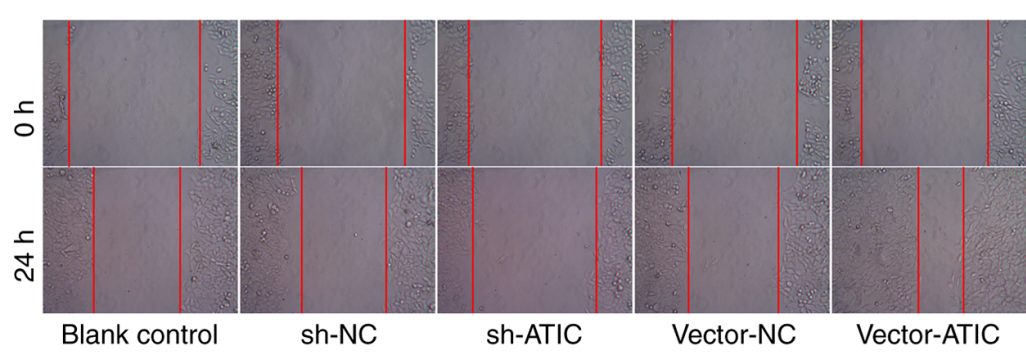

E

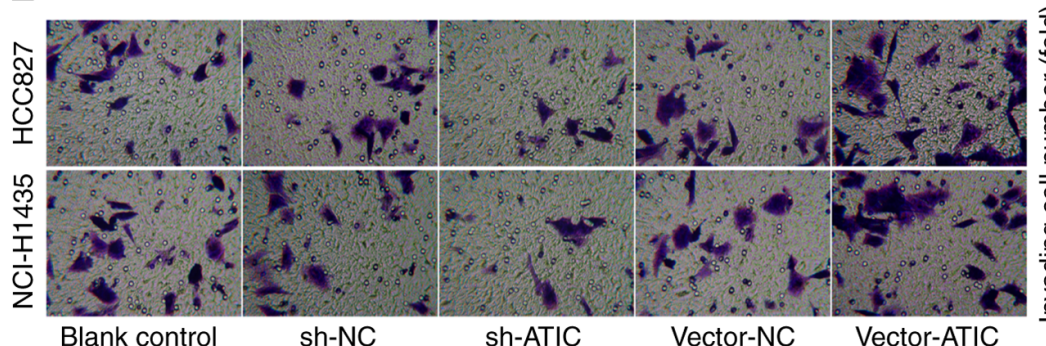

B

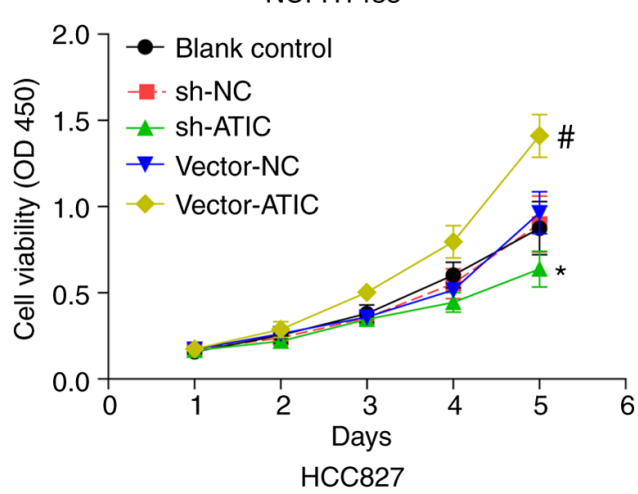

$\mathrm{NCl}-\mathrm{H} 1435$
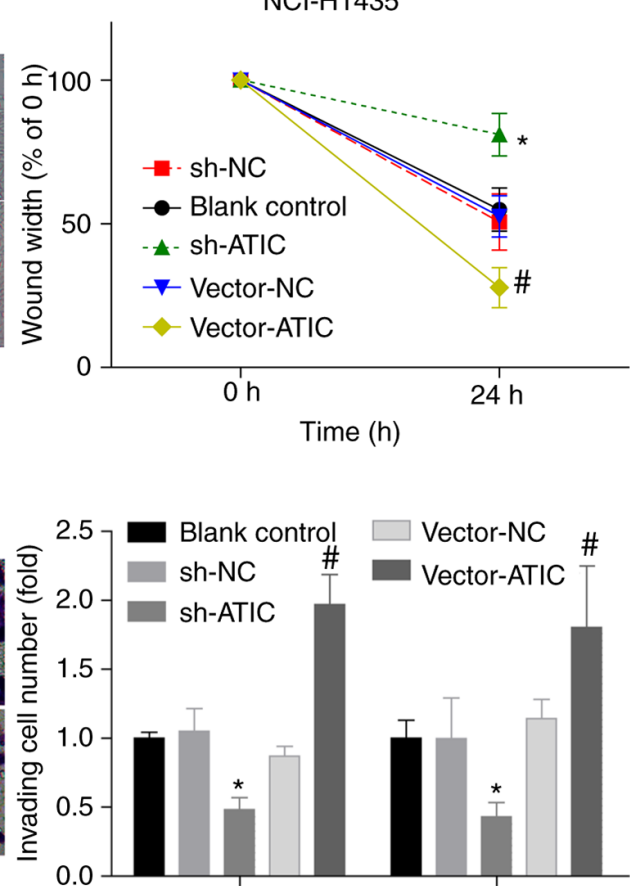

HCC827

$\mathrm{NCl}-\mathrm{H} 1435$

Figure 3. ATIC promotes LUAD cell growth and migration. (A and B) Cell growth ability in the blank control (without treatment), sh-NC, sh-ATIC, vector-NC and vector-ATIC groups was assessed using CCK-8 assay. (C and D) Cell migratory ability in the blank control, sh-NC, sh-ATIC, vector-NC and vector-ATIC groups was assessed using wound healing assay. (E) Cell invasive ability in the blank control (without treatment), sh-NC, sh-ATIC, vector-NC and vector-ATIC groups was assessed using Transwell assay. ${ }^{*} \mathrm{P}<0.05$, vs. sh-NC group; ${ }^{\#} \mathrm{P}<0.05$, vs. vector-NC group. ATIC, 5-aminoimidazole-4-carboxamide ribonucleotide formyltransferase/IMP cyclohydrolase; LUAD, lung adenocarcinoma.

its higher knockdown efficiency, in comparison with sh-ATIC-1. The growth (Fig. 3A and B), migratory (Fig. 3C and D) and invasive (Fig. 3E) abilities of the HCC827 and NCI-H1435 cells were enhanced by $\sim 50-100 \%$ when ATIC was overexpressed, whereas this effect was attenuated by $\sim 50 \%$ when ATIC was silenced (Fig. 3A and E). These findings illustrated that ATIC promoted cell growth and migratory abilities in LUAD.

ATIC positively modulates Myc expression in LUAD cells. In order to elucidate the mechanism through which ATIC 
A

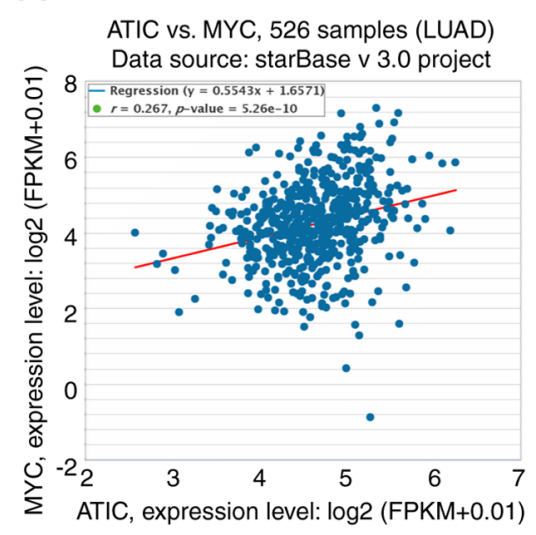

B

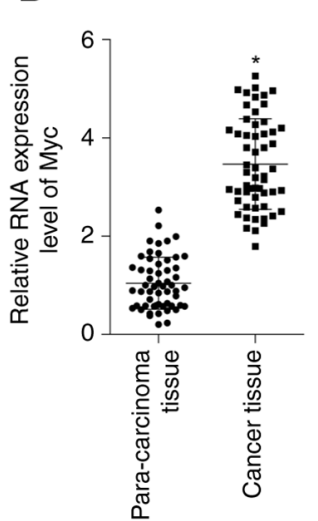

C

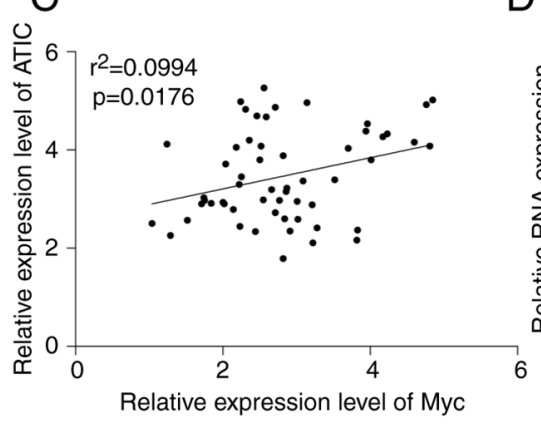

E

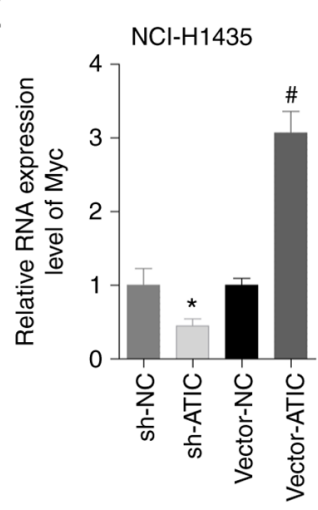

I

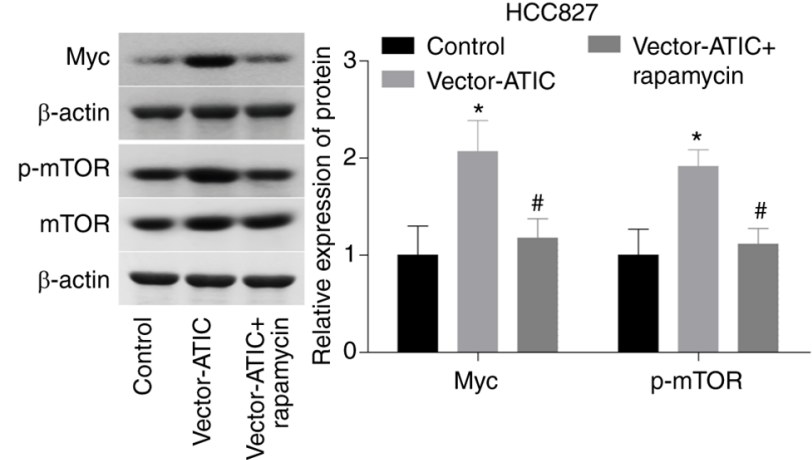

$\mathrm{F}$

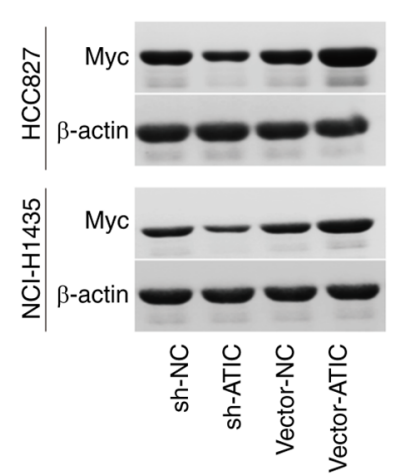

G

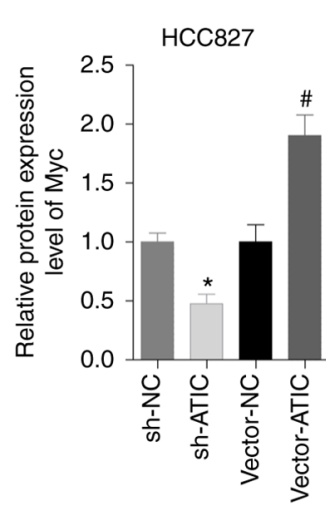

$J$

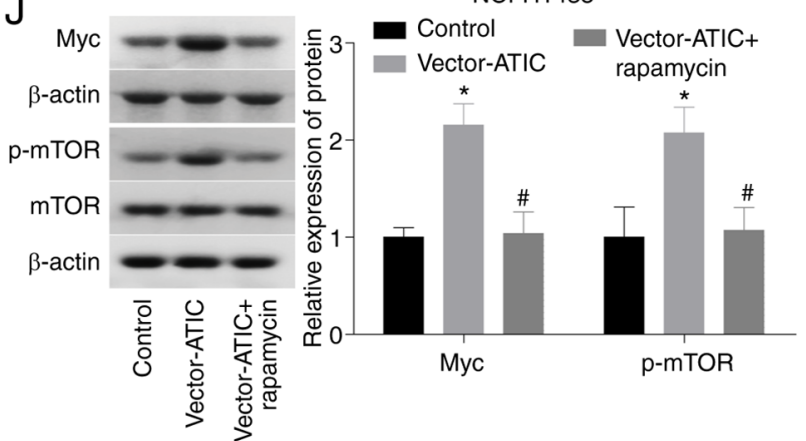

$\mathrm{K}$

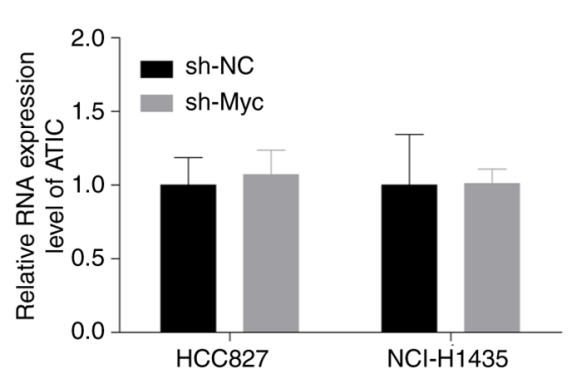

$\mathrm{L}$

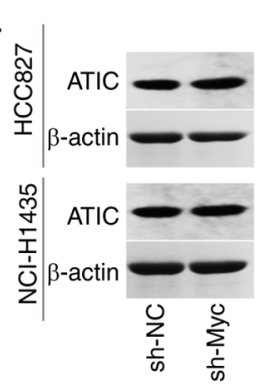

$\mathrm{H} \quad \mathrm{NCl}-\mathrm{H} 1435$

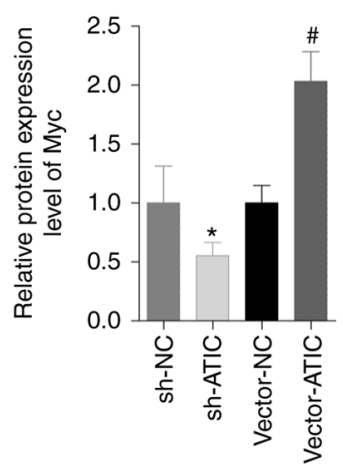

NCl-H1435

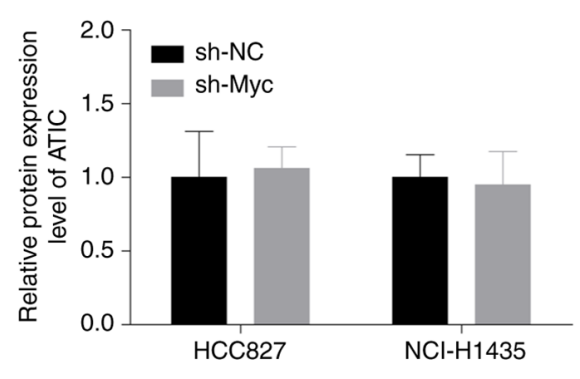

Figure 4. ATIC increases Myc expression in LUAD cells. (A) StarBase was used to assess the association between ATIC and Myc expression levels in LUAD. (B) Myc levels in 56 LUAD tissues were determined using RT-qPCR. (C) Pearson's correlation analysis was applied to evaluate the association between the expression levels of ATIC and Myc in LUAD cases. Myc expression at the mRNA and protein levels was determined using (D and E) RT-qPCR and (F-H) western blot analysis in HCC827 and NCI-H1435 cells. (I and J) Western blot analysis was used to examine the expression of Myc, mTOR and p-mTOR in the different groups of HCC827 and NCI-H1435 cells. (K and L) The mRNA and protein levels of ATIC were determined using RT-qPCR and western blot analysis when Myc expression was silenced in the HCC 827 and NCI-H1435 cells. " $\mathrm{P}<0.05$, vs. sh-NC group; "P<0.05, vs. vector-NC group. ATIC, 5-aminoimidazole-4-carboxamide ribonucleotide formyltransferase/IMP cyclohydrolase; LUAD, lung adenocarcinoma; p-mTOR, phosphorylated mTOR; RT-qPCR, reverse transcription-quantitative PCR.

promotes LUAD progression, the association between ATIC and Myc was subsequently evaluated in LUAD. According to the StarBase analysis results, a weak association between the ATIC and Myc expression levels in LUAD tissues was observed (Fig. 4A). Additionally, the Myc mRNA expression levels were also analysed in the 56 paired LUAD tissues and 
normal tissues. The results demonstrated that Myc expression was elevated in LUAD, while a significant positive correlation was observed with the ATIC levels in the LUAD cases (Fig. 4B and C). Subsequently, the effect of ATIC on Myc expression in the HCC827 and NCI-H1435 cells was evaluated. The silencing of ATIC led to a decrease in Myc expression, while the overexpression of ATIC resulted in an increase in Myc expression levels (Fig. 4D and H). It has been reported that ATIC can activate the mTOR pathway which modulates Myc expression $(12,17,18)$; thus, it was hypothesized that the increased expression of Myc induced by ATIC may be related to mTOR activation. As was expected, ATIC overexpression increased the expression of p-mTOR and Myc, which was reversed by rapamycin, an mTOR inhibitor (Fig. 4I and J). Additionally, it was assessed whether Myc modulates ATIC expression using qPCR and western blot analysis. Transfection with sh-Myc- 2 led to an $80 \%$ decrease in the Myc mRNA levels and a $60 \%$ decrease in the Myc protein levels in the HCC827 and NCI-H1435 cells (Fig. S1E and F). However, the ATIC mRNA and protein levels did not exhibit an obvious change when Myc was silenced in the HCC827 and NCI-H1435 cells (Fig. 4K and L). These results thus demonstrated that ATIC positively modulated Myc expression in LUAD cells.

ATIC promotes cell growth and migration in a Myc-dependent manner. It was then examined whether Myc was involved in the ATIC-mediated LUAD progression by using rescue experiments. The growth, migratory and invasive abilities of the HCC827 and NCI-H1435 cells were decreased $\sim 0.5$-fold when Myc was silenced, in comparison with the control group. Additionally, the silencing of Myc abolished the ability of ATIC to promote the growth (Fig. 5A and B), migration (Fig. 5C and D) and invasion (Fig. 5E) of the HCC827 and NCI-H1435 cells. These results confirmed that ATIC promoted LUAD cell growth and migration in a Myc-dependent manner.

\section{Discussion}

The main aim of the present study was to elucidate the role of ATIC in LUAD progression. The results demonstrated that ATIC expression was increased in LUAD tissues and cells, and that the high expression of ATIC was associated with malignant clinical phenotypes and lower survival rates of patients with LUAD. Additionally, it was revealed that the overexpression of ATIC significantly promoted cell growth viability, migration and invasion by increasing Myc expression in LUAD.

ATIC is a $64 \mathrm{kDa}$ bifunctional enzyme that modulates the activities of two enzymes in the de novo purine biosynthesis pathway, AICAR and IMP cyclohydrolase $(20,21)$. Previous studies have demonstrated that the de novo purine biosynthesis pathway is closely implicated in the occurrence and development of various types of cancer. For example, Lv et al (7) found that de novo nucleotide synthesis was increased in metastatic breast cancer cells. In addition, blocking de novo synthesis with phosphoribosyl pyrophosphate synthetase 2 (PRPS2) downregulation has been shown to result in the marked inhibition of cell stemness and lung metastasis (22). The inhibition of AICAR activity can cooperate with pemetrexed to suppress tumour growth (22). As an enzyme that regulates the de novo purine biosynthesis pathway, ATIC has also been reported to be involved in carcinogenesis. Park and Shin (23) found that the polymorphism of ATIC $(347 \mathrm{C}>\mathrm{G})$ may be a factor affecting the response to methotrexate in osteosarcoma. Li et al (12) demonstrated that ATIC was highly expressed in HCC tissues and that the high expression of ATIC was related to a poor prognosis; ATIC downregulation suppresses cell proliferation, colony formation and migration by modulating the adenosine monophosphate-activated protein kinase (AMPK)/mTOR pathway. By using bioinformatics analysis, Zhu et al (24) identified that ATIC was a risk factor for LUAD that may present a high potential in predicting the survival rates of patients with LUAD. Herein, it was demonstrated for the first time, to the best of our knowledge, that the expression of ATIC was markedly higher in LUAD tissues compared with normal lung tissues. In addition, it was demonstrated that the higher expression of ATIC was closely associated with advanced TNM stages, higher lymph node metastasis rates, a poorer tissue differentiation and lower survival rates. Moreover, ATIC expression levels in three LUAD cell lines (HCC827, NCI-H1435 and HCC4006) were assessed and it was observed that the ATIC levels were increased in LUAD cells, in comparison with normal lung epithelial BEAS-2B cells. The HCC827 and NCI-H1435 cells demonstrated moderate ATIC expression levels in comparison with the HCC4006 and BEAS-2B cells; thus, gain- and loss-of-function assays were performed using these two cell lines, in order to assess the effect of ATIC on LUAD progression in vitro. The results demonstrated that ATIC overexpression led to the promotion of cell growth, migration and invasion, while ATIC silencing inversely lead to the suppression of cell growth, migration and invasion. The results of the present study revealed that ATIC functioned as an oncogene in LUAD.

It was observed that ATIC expression positively correlated with Myc expression in the LUAD cases. It has been demonstrated that Myc is an oncogene in LUAD and that a high level of Myc is closely linked to a decreased survival rate of patients with LUAD $(25,26)$. Through the modulation of Myc expression, a number of genes have been reported to be involved in the occurrence and development of LUAD. For instance, lysophosphatidylcholine acyltransferase 1 has been reported to promote the brain metastasis of LUAD by activating the PI3K/AKT/Myc pathway (27). miR-1827 has been reported to inhibit tumour growth by reducing Myc and Family with sequence similarity 83 member $F$ gene levels in LUAD (28). Additionally, Myc has been identified to vitally contribute in facilitating nucleotide biosynthesis by increasing the expression of the nucleotide synthesis enzyme PRPS2 (29) and phosphoribosylaminoimidazole carboxylase (30). In the present study, it was revealed that ATIC overexpression increased Myc expression, whereas Myc did not affect ATIC expression in LUAD cells. Since Myc is a downstream factor of the mTOR pathway that can be activated by $\operatorname{ATIC}(12,17,18)$, it was hypothesized that the increased expression of Myc induced by ATIC may be related to mTOR activation. This was verified by using western blot analysis; rapamycin stimulation reversed the ATIC-mediated increase in Myc expression.

In order to reveal whether Myc is involved in ATICmediated LUAD progression, rescue experiments were also performed. As was expected, the results demonstrated that 
A

HCC827

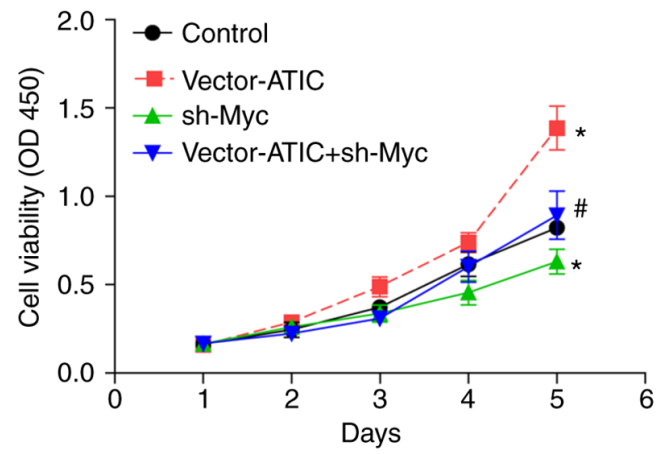

C

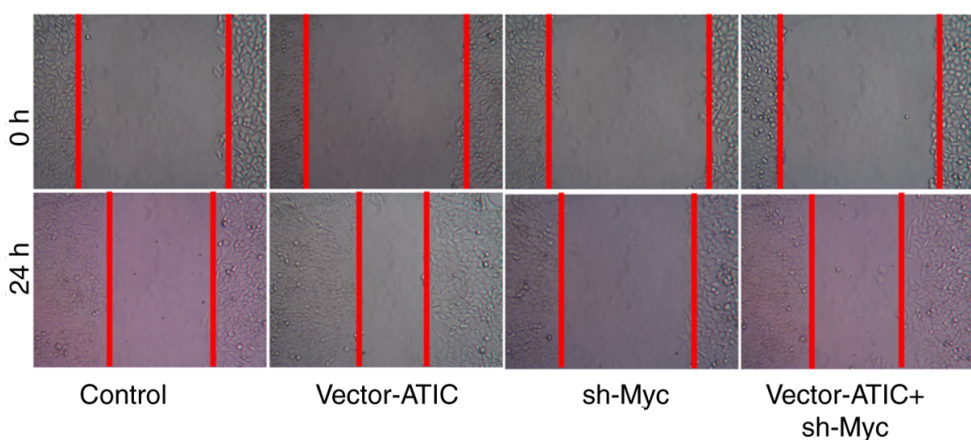

D

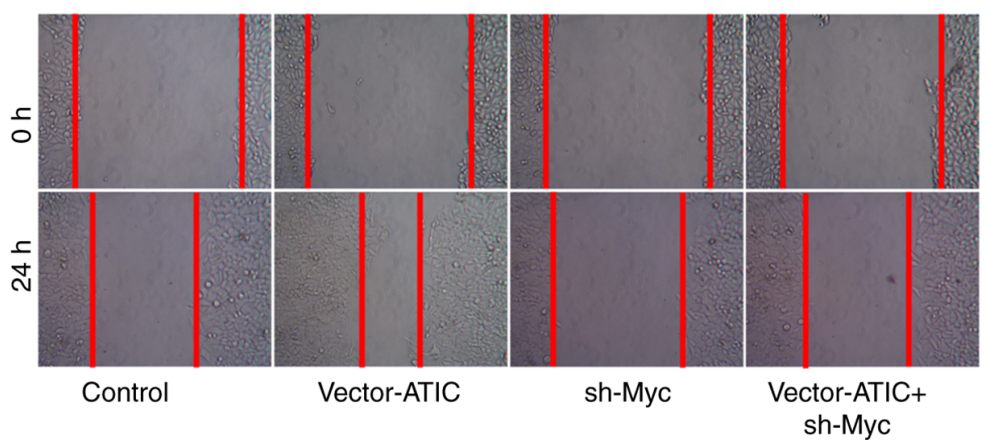

$\mathrm{NCl}-\mathrm{H} 1435$

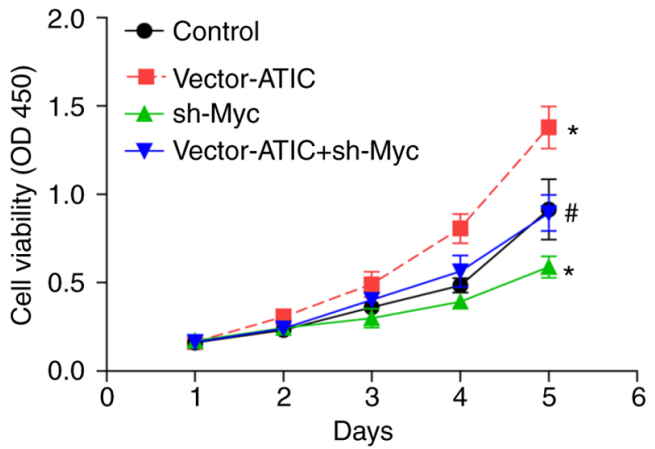

HCC827

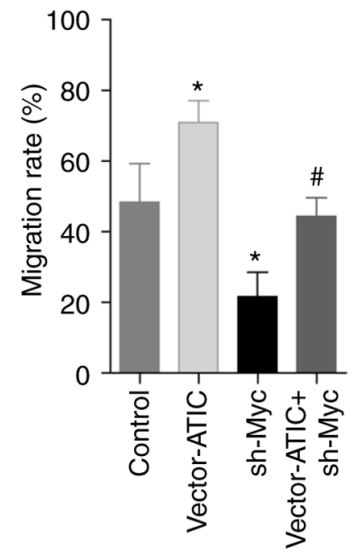

$\mathrm{NCl}-\mathrm{H} 1435$

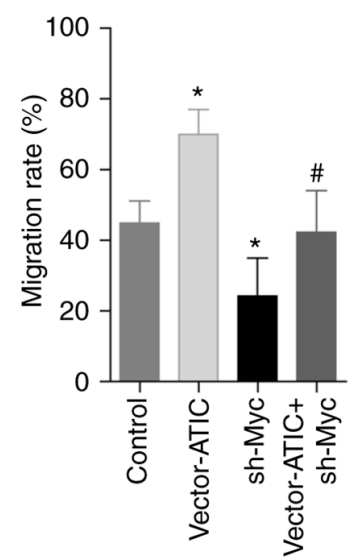

E
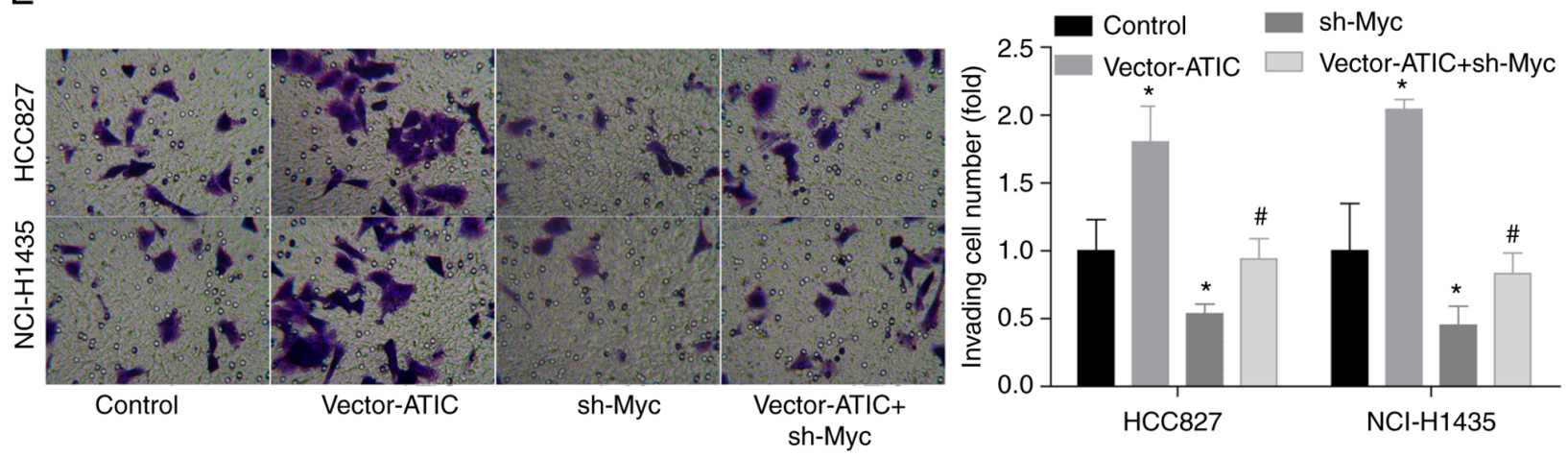

Figure 5. ATIC promotes cell growth and migration in a Myc-dependent manner. (A and B) Cell growth ability in the control, vector-ATIC, sh-Myc and vector-ATIC + sh-Myc groups was measured using CCK-8 assay. (C and D) Cell migration ability in the control, vector-ATIC, sh-Myc and vector-ATIC + sh-Myc groups was assessed using wound healing assay. (E) Cell invasive ability in the control, vector-ATIC, sh-Myc and vector-ATIC + sh-Myc groups was assessed using Transwell chambers. ${ }^{*} \mathrm{P}<0.05$, vs. control group; ${ }^{*} \mathrm{P}<0.05$, vs. vector-ATIC group. ATIC, 5 -aminoimidazole-4-carboxamide ribonucleotide formyltransferase/IMP cyclohydrolase; LUAD, lung adenocarcinoma. 
the silencing of Myc attenuated the promotion of cell growth, migration and invasion induced by ATIC, suggesting that ATIC promoted LUAD cell growth and migration by increasing Myc expression.

Several limitations should be underlined for the present study. The roles of ATIC/Myc in tumour formation in vivo and the drug resistance of LUAD were not explored. Thus, the authors intend to investigate these parameters in future studies. Furthermore, the mutation status of EGFR, anaplastic lymphoma kinase and proto-oncogene tyrosine-protein kinase ROS in clinical samples were not assessed, as well as their associations with ATIC expression.

In conclusion, it was revealed that ATIC was highly expressed in LUAD tissues and cells, and that the high expression of ATIC was closely associated with lower survival rates and an advanced clinical stage of patients with LUAD. The overexpression of ATIC significantly promoted cell growth, migration and invasion by increasing Myc expression in LUAD. The findings of the present study may provide a possible novel diagnostic marker and treatment target for LUAD.

\section{Acknowledgements}

Not applicable.

\section{Funding}

No funding was received.

\section{Availability of data and materials}

The datasets used and/or analysed during the current study are available from the corresponding author on reasonable request.

\section{Authors' contributions}

NN conceived the study performed the experiments and revised the manuscript. JZ, XK and $\mathrm{WZ}$ performed western blotting and RT-qPCR experiments and wrote the manuscript. CF, SL, JF and YY analysed the data. All authors have read and approved the final manuscript. NN and JZ confirm the authenticity of all the raw data.

\section{Ethics approval and consent to participate}

The present study was approved by the Ethics Committee of National Cancer Center/National Clinical Research Center for Cancer/Cancer Hospital and Shenzhen Hospital Chinese Academy of Medical Sciences and Peking Union Medical College prior to this study (approval no. KYLX2012-107). Signed written informed consent was acquired by all patients or their families.

\section{Patient consent for publication}

Not applicable.

\section{Competing interests}

The authors declare that they have no competing interests.

\section{References}

1. Sung H, Ferlay J, Siegel RL, Laversanne M, Soerjomataram I, Jemal A and Bray F: Global cancer statistics 2020: GLOBOCAN estimates of incidence and mortality worldwide for 36 cancers in 185 countries. CA Cancer J Clin 71: 209-249, 2021.

2. Herbst RS, Morgensztern D and Boshoff C: The biology and management of non-small cell lung cancer. Nature 553: 446-454, 2018.

3. Xu Y, Jiang Q, Liu H, Xiao X, Yang D, Saw PE and Luo B: DHX37 impacts prognosis of hepatocellular carcinoma and lung adenocarcinoma through immune infiltration. J Immunol Res 2020: 8835393, 2020.

4. Xu Y, Liu Z, Li H, Feng S, Li Q, Li J and Li S: VEGI downregulation is correlated with nodal metastasis and poor prognosis in lung adenocarcinoma. Mol Clin Oncol 14: 25, 2021.

5. Cai J, Deng H, Luo L, You L, Liao H and Zheng Y: Decreased expression of JAK1 associated with immune infiltration and poor prognosis in lung adenocarcinoma. Aging (Albany NY) 13: 2073-2088, 2020.

6. Robinson AD, Eich ML and Varambally S: Dysregulation of de novo nucleotide biosynthetic pathway enzymes in cancer and targeting opportunities. Cancer Lett 470: 134-140, 2020.

7. Lv Y, Wang X, Li X, Xu G, Bai Y, Wu J, Piao Y, Shi Y, Xiang R and Wang L: Nucleotide de novo synthesis increases breast cancer stemness and metastasis via cGMP-PKG-MAPK signaling pathway. PLoS Biol 18: e3000872, 2020.

8. Fan TWM, Bruntz RC, Yang Y, Song H, Chernyavskaya Y, Deng P, Zhang Y, Shah PP, Beverly LJ, Qi Z, et al: De novo synthesis of serine and glycine fuels purine nucleotide biosynthesis in human lung cancer tissues. J Biol Chem 294: 13464-13477, 2019.

9. Yamaoka T, Kondo M, Honda S, Iwahana H, Moritani M, Ii S, Yoshimoto K and Itakura M: Amidophosphoribosyltransferase limits the rate of cell growth-linked de novo purine biosynthesis in the presence of constant capacity of salvage purine biosynthesis. J Biol Chem 272: 17719-17725, 1997.

10. VermaP,KarB, Varshney R, Roy Pand Sharma AK: Characterization of AICAR transformylase/IMP cyclohydrolase (ATIC) from Staphylococcus lugdunensis. FEBS J 284: 4233-4261, 2017.

11. Li R, Chen G, Dang Y, He R, Liu A, Ma J and Wang C: Upregulation of ATIC in multiple myeloma tissues based on tissue microarray and gene microarrays. Int J Lab Hematol 43: 409-417, 2021

12. Li M, Jin C, Xu M, Zhou L, Li D and Yin Y: Bifunctional enzyme ATIC promotes propagation of hepatocellular carcinoma by regulating AMPK-mTOR-S6 K1 signaling. Cell communication and signaling. Cell Commun Signal 15: 52, 2017.

13. Hsieh AL, Walton ZE, Altman BJ, Stine ZE and Dang CV: MYC and metabolism on the path to cancer. Semin Cell Dev Biol 43: 11-21, 2015

14. Dang CV: MYC on the path to cancer. Cell 149: 22-35, 2012.

15. Ma L, Young J, Prabhala H, Pan E, Mestdagh P, Muth D, Teruya-Feldstein J, Reinhardt F, Onder TT, Valastyan S, et al: miR-9, a MYC/MYCN-activated microRNA, regulates E-cadherin and cancer metastasis. Nat Cell Biol 12: 247-256, 2010.

16. Zack TI, Schumacher SE, Carter SL, Cherniack AD, Saksena G, Tabak B, Lawrence MS, Zhsng CZ, Wala J, Mermel CH, et al: Pan-cancer patterns of somatic copy number alteration. Nat Genet 45: 1134-1140, 2013.

17. Shen P, Reineke LC, Knutsen E, Chen M, Pichler M, Ling H and Calin GA: Metformin blocks MYC protein synthesis in colorectal cancer via mTOR-4EBP-eIF4E and MNK1-eIF4G-eIF4E signaling. Mol Oncol 12: 1856-1870, 2018.

18. Xu D, Xie R, Xu Z, Zhao Z, Ding M, Chen W, Zhang J, Mao E, Chen E, Chen Y, et al: mTOR-Myc axis drives acinar-to-dendritic cell transition and the $\mathrm{CD} 4^{+} \mathrm{T}$ cell immune response in acute pancreatitis. Cell Death Dis 11: 416, 2020.

19. Livak KJ and Schmittgen TD: Analysis of relative gene expression data using real-time quantitative PCR and the 2(-Delta Delta C(T)) method. Methods 25: 402-408, 2001.

20. Greasley SE, Horton P, Ramcharan J, Beardsley GP, Benkovic SJ and Wilson IA: Crystal structure of a bifunctional transformylase and cyclohydrolase enzyme in purine biosynthesis. Nat Struct Biol 8: 402-406, 2001

21. Vergis JM, Bulock KG, Fleming KG and Beardsley GP: Human 5-aminoimidazole-4-carboxamide ribonucleotide transformylase/inosine 5'-monophosphate cyclohydrolase. A bifunctional protein requiring dimerization for transformylase activity but not for cyclohydrolase activity. J Biol Chem 276: 7727-7733, 2001. 
22. Racanelli AC, Rothbart SB, Heyer CL and Moran RG: Therapeutics by cytotoxic metabolite accumulation: Pemetrexed causes ZMP accumulation, AMPK activation, and mammalian target of rapamycin inhibition. Cancer Res 69: 5467-5474, 2009.

23. Park JA and Shin HY: ATIC gene polymorphism and histologic response to chemotherapy in pediatric osteosarcoma. J Pediatr Hematol Oncol 39: e270-e274, 2017.

24. Zhu J, Wang M and Hu D: Development of an autophagy-related gene prognostic signature in lung adenocarcinoma and lung squamous cell carcinoma. PeerJ 8: e8288, 2020.

25. Iwakawa R, Kohno T, Kato M, Shiraishi K, Tsuta K, Noguchi M, Ogawa S and Yokota J: MYC amplification as a prognostic marker of early-stage lung adenocarcinoma identified by whole genome copy number analysis. Clin Cancer Res 17: 1481-1489, 2011.

26. Ciribilli Y and Borlak J: Oncogenomics of c-Myc transgenic mice reveal novel regulators of extracellular signaling, angiogenesis and invasion with clinical significance for human lung adenocarcinoma. Oncotarget 8: 101808-101831, 2017.
27. Wei C, Dong X, Lu H, Tong F, Chen L, Zhang R, Dong J, Hu Y, Wu G and Dong X: LPCAT1 promotes brain metastasis of lung adenocarcinoma by up-regulating PI3K/AKT/MYC pathway. J Exp Clin Cancer Res 38: 95, 2019.

28. Fan G, Xu P and Tu P: miR-1827 functions as a tumor suppressor in lung adenocarcinoma by targeting MYC and FAM83F. J Cell Biochem 121: 1675-1689, 2020.

29. Cunningham JT, Moreno MV, Lodi A, Ronen SM and Ruggero D: Protein and nucleotide biosynthesis are coupled by a single rate-limiting enzyme, PRPS2, to drive cancer. Cell 157: 1088-1103, 2014

30. Barfeld SJ, Fazli L, Persson M, Marjavaara L, Urbanucci A, Kaukoniemi KM, Rennie PS, Ceder Y, Chabes A, Visakorpi T and Mills IG: Myc-dependent purine biosynthesis affects nucleolar stress and therapy response in prostate cancer. Oncotarget 6: $12587-12602,2015$.

(i) $\Theta$ This work is licensed under a Creative Commons Attribution-NonCommercial-NoDerivatives 4.0 International (CC BY-NC-ND 4.0) License. 